\title{
Prototyping Framework for SDL with Evolving Semantics
}

\author{
Peter Csurgay \\ Department of Telematics, Norwegian University of Science and Technology, clo Traffic \\ Analysis and Network Performance Laboratory, Ericsson Telecommunications Ltd, \\ Address: Laborc utca 1, Budapest 1037, Hungary, Phone: (+36 20) 9793049 \\ E-mail: csurgay@item.ntnu.no
}

Key words: SDL, prototyping, formal semantics, extension of FDTs, Java technology

Abstract: The definition of the ITU-T Specification and Description Language (SDL) evolves, its semantic interpretation and modelling features are subject to modifications and extensions to meet demands of new application domains and new modelling contexts. Tools are slow in following this evolution, although automated support is vital in applying formal methods for the design and evaluation of telecommunications protocols. This paper introduces a prototyping framework that supports traditional and new tool functions for specifications in SDL with evolving semantics, thus eases the evolution process as well. The framework includes automatic parser generation and abstract grammar definition from Z.100-style EBNF descriptions, and an easily extensible object-oriented formal definition of the semantic interpretation model. Java technology has been used to implement an open framework of distributed visualisation, control, and evaluation components. Early implementation of the framework was applied to prototyping systems with extended SDL semantics in the performance evaluation context.

\section{INTRODUCTION}

Initially, SDL was meant for unambiguous specification and description of telecommunications systems. As Z.100 states: 'specification' of a system is the description of its required behaviour, and 'description' is the 
description of its actual behaviour [5]. Recently, however, SDL has been used in various other contexts, like performance evaluation or automatic implementation of formally specified systems. Behaviour of an SDL system is of non-deterministic nature, not just because it has language constructs to express non-determinism on process level, but because it is composed of asynchronously communicating concurrent extended finite state machines (CEFSMs). This non-determinism is the basis of modelling concurrent entities of the real world, and also of specifying and validating a set of possible traces in a concise manner. Time and ordering of internal events plays key role in SDL's non-determinism. Due to the vague time semantics of SDL (signal transactions and transitions may or may not take time), tool builders and users of SDL choose legal, but different interpretations of time semantics when simulating or interpreting SDL systems. A legal interpretation for example (most probably inspired by the way some leading tools handle advancing of time) is that time passes only if there are no armed transitions (no valid input signals in queues) of processes. This interpretation is legal, even reduces the set of possible traces simplifying e.g. validation, but not appropriate for e.g. performance modelling [12]. A prototyping framework should support generation of possible traces of system behaviour based on different interpretation models. This is achieved by separation of different interpretation functions in a modular object-oriented model of formal semantics. Due to the unsatisfying formal semantic definition of Z.100 for new application domains various modifications and extensions to SDL's semantics [10,13], and even new formal semantic interpretations emerge [11]. (These improvements will influence the recommendation, and eventually, a new version SDL-2000 might include modifications [7].) Current tools are not open in the sense that they employ a specific language definition and specific semantic interpretation with little room for experimentation with them. A prototyping framework should support the evolution process and protocol engineering with evolved SDL semantics by taking formal semantics and interpretation models along with syntax and system specification as input to the prototyping process.

The ITU-T Recommendation Z.100 defines the Specification and Description Language (SDL) with (1) its concrete grammars SDL/PR and SDL/GR, (2) an abstract syntax AS1 equivalent to the concrete grammars, (3) rules to transform a system specification into abstract syntax, and (4) the SDL-machine semantics to interpret a specification given in terms of the abstract syntax. The structure of the definition that eventually leads to the behaviour interpretation of SDL systems and the effect of the evolution of SDL semantics on it is illustrated on Figure 1. (1) is given by an extended Backus-Naur Form (EBNF) notation. (2) uses a subset of Meta IV. (3) and 
(4) is given both informally and formally in the Annex F to Z.100 as the static and dynamic semantics respectively. On over 500 pages, it defines a set of Meta IV (a formal language based on synchronously communicating concurrent processes) programs for static correctness analysis, transformation of concrete syntax into abstract syntax with removal/substitution of several language constructs, and interpretation of behaviour the SDL-machine. The Annex F formal model does not help human understanding of SDL's semantics simply because its size, and Z.100 gives precedence to the informal definition.

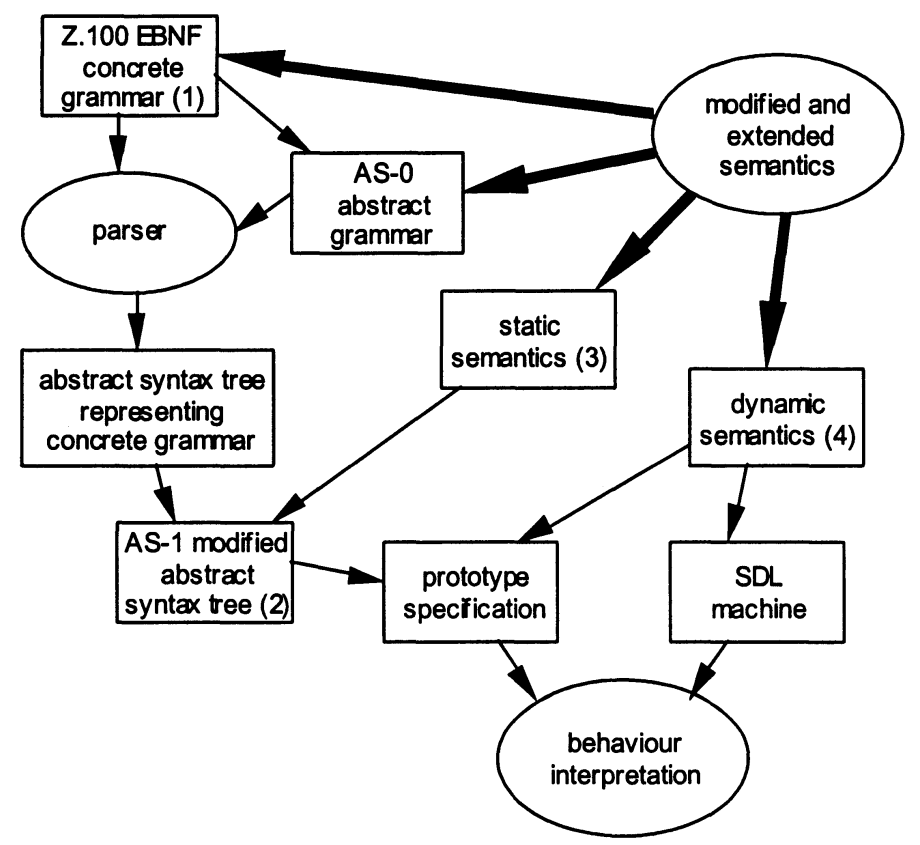

Figure 1. Effect of evolving semantics on Z.100 SDL definition

Because of the evolutionary nature of SDL, a prototyping framework shall support semi-automatic processing for (1), support for formal definition of semantic actions and semi-automatic generation of (2), a concise and flexible formal definition of (3), an extensible formal interpretation model for (4), and finally, a portable, open interpretation platform for visualisation, control and evaluation of systems specified in SDL with extended semantics (Figure 2). 
specification domain

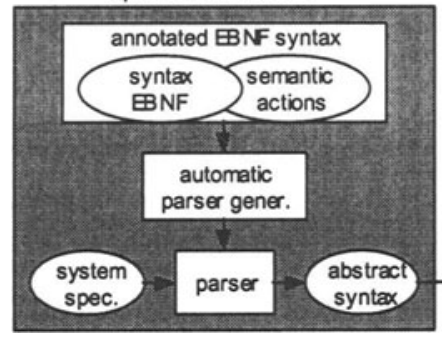

instance domain

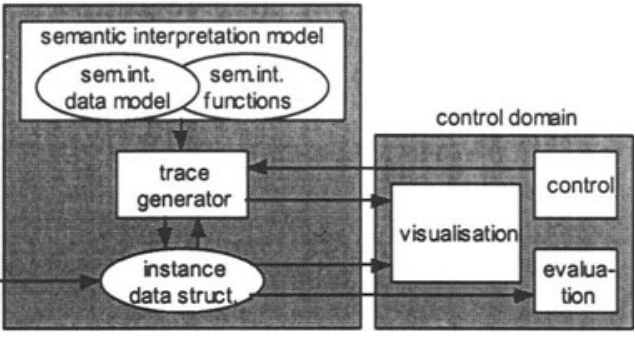

Figure 2. Functions and data structures of the prototyping framework

Section 2 describes the automatic parser and abstract syntax tree generation process. Section 3 shows the new approaches in the objectoriented semantic interpretation model with examples from the specification and instance domains. Section 4 illustrates the application of the framework to specifications in SDL with extended semantics in the performance evaluation context. Two different packet transfer protocol alternatives were modelled in PerfSDL (a semantic extension to SDL [10]), and they were compared by performance evaluation based on performance traces produced by a prototype interpretation model. The paper concludes in Section 5 .

\section{AUTOMATIC PARSER GENERATION}

The traditional or most understood ways of translating a sentence of a formal language to a sentence of another follow the analysis-synthesis model of compilation. The analysis part breaks up the source program into constituent pieces (tokenising or lexical analysis), and creates an intermediate representation (parsing or syntax analysis). The synthesis part constructs the target language sentence from this intermediate representation. While the analysis part has well settled methods and algorithms [8], the synthesis part requires specialised techniques. Lexical analysers can be easily built from the lexical rules of a language, and it is most often done following rather intuitive than automated or formal procedures. The aim of syntax analysis is to generate the intermediate representation (often an abstract syntax tree) of the source sentence from its tokenised form. This Section will focus on the syntax analysis part, while Section 3 and 4 illustrates the synthesis part through examples. 


\subsection{Parsing the BNF grammar of Z.100}

The recommendation contains the specification of both the graphical (SDL-GR) and textual (SDL-PR) form of the language in a mixed dual fashion having <sdl specification> the common root symbol. The project presented in this paper only processes the textual form, i.e. the grammar is taken to be the set of rules of the SDL-PR definition without the phrases referring to graphical elements of SDL-GR. To be able to automatically process the BNF definition given by Z.100, first a tokenizer, parser, and abstract syntax tree have to be built. For reasons mentioned above, an automated parser generation approach was chosen based on the grammar's BNF definition directly derived from the text of the $\mathrm{Z} .100$ recommendation. The recommendation uses an extension of the original Backus Naur Form notation, however, it does not follow the "ISO/IEC 14977 Extended BNF" standard for the extensions. The following meta-BNF grammar (using the very same style BNF) can be derived from the syntactic meta-rules given by Z.100 informally. Semantics of the notation is given in Table 1.

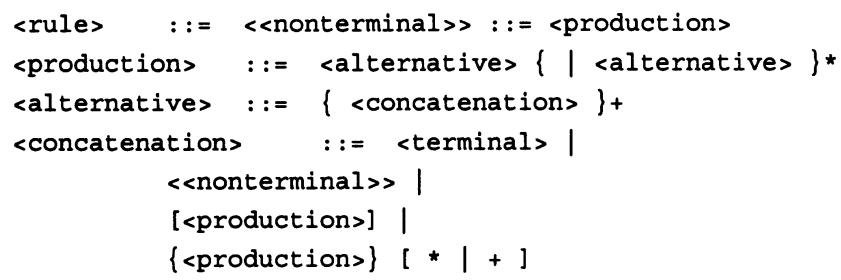

Table 1. Semantics of BNF extensions in Z.100

\begin{tabular}{|c|c|}
\hline$::=$ & Defining symbol of rules \\
\hline$<>$ & Enclosing nonterminals \\
\hline 1 & Separating alternatives \\
\hline$[1]$ & Enclosing optionals; zero or one occurrence \\
\hline\{\} & Enclosing groups; one occurrence \\
\hline\{\}$^{*}$ & $\begin{array}{l}\begin{array}{l}\text { Enclosing repetition; zero or more } \\
\text { occurrences }\end{array} \\
\text { oct }\end{array}$ \\
\hline\{\}$^{+}$ & $\begin{array}{l}\text { Enclosing repetition; one or more } \\
\text { occurrences }\end{array}$ \\
\hline
\end{tabular}

This small grammar will be the basis for all further automatic parser generation issues. Note, that for each rule of this grammar the first terminal symbol (lookahead) unambiguously determines which branch to parse on. Therefore a simple predictive parser (that is a recursive descent parser with 
no backtracking) can syntax analyse its language (i.e. the BNF rules of Z.100).

\subsection{Abstract syntax tree for Z.100}

According to the BNF grammar above and the semantics of the metadescription in Z.100, node types for the abstract syntax tree was chosen. These node types and their relation for the valid branching topologies are illustrated in Figure 3. For each node type there is a Java class that is capable of predictive parsing the Z.100 BNF input stream, and building on the abstract syntax tree according to the fired rules.

Given the complete abstract syntax tree of the Z.100 BNF definition, several interesting observations can be made. For instance by producing a pretty print of all rules, and comparing them to the original definition, metainformation can be derived about the recommendations and the algorithm. The Z.100 document Addendum 1 gives of list of changes and corrections to the recommendation. Contact the author for a list of bugs not covered by the Addendum, that were made during formal processing of the Z.100 recommendation.

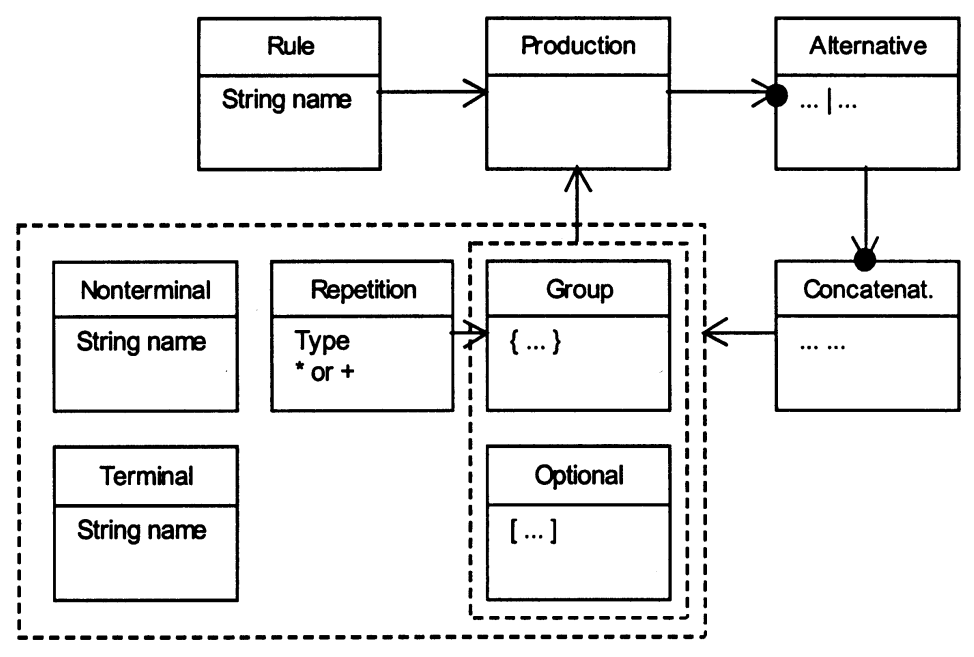

Figure 3. Z.100 BNF abstract syntax tree node types and their branching 


\subsection{Recursive descent parsing with backtracking}

The most common method to syntax analyse formal languages given by context-free grammars is to pre-process the grammar first (e.g. convert it to Greibach Normal Form), and construct a parser of linear complexity (e.g. predictive parser). In this project however, to preserve local integrity of rules of our original BNF grammar for reasons explained earlier, we decided to generate a recursive descent parser for SDL. Therefore this method is shortly introduced here. In the family of top-down parsers, the most intuitive (or more importantly for our reasons, the closest following a context free grammar in structure) is the recursive descent parser. Note, that not all context free grammars can be analysed with recursive descent parsers (left recursive grammars leads them into infinite looping), but the grammar in $\mathrm{Z} .100$ is in a proper form in this sense. In recursive descent parsers a logical value returning procedure is associated with each nonterminal symbol. These procedures return true if the input matches their terminal symbols and the procedures associated with their nonterminal symbols also returned true, and false in any other case. These procedures might call each other recursively, implementing an implicit stack automaton needed to analyse context free languages in classical theory of formal languages. Table 2 explains the mapping from BNF constructs and operation of logical values generated by terminal and nonterminal symbols of a rule.

Table 2. Logical operations for BNF constructs in recursive descent parsing

\begin{tabular}{|l|c|l|}
\hline Alternative & $\mathrm{x} \mid \mathrm{y}$ & If $\mathrm{x}$ or $\mathrm{y}$ then true else false \\
\hline Concatenation & $\mathrm{x} \mathrm{y}$ & If $\mathrm{x}$ and $\mathrm{y}$ then true else false \\
\hline Optional & {$[\mathrm{x}]$} & If $\mathrm{x}$; true \\
\hline Group & \{\} & Parentheses in logical expressions \\
\hline Repetition & $\{\mathrm{x}\}^{*}$ & While $\mathrm{x}$; true \\
\hline Repetition & $\{\mathrm{x}\}+$ & If $\mathrm{x}$ then while $\mathrm{x} ;$ true else false \\
\hline
\end{tabular}

Beside the advantages of this way of parsing (i.e. no modification to the BNF rules are needed and semantic constraints can easily be applied to the generated abstract syntax tree), there is one serious inconvenience. There is no problem until all procedures (implementing rules of the original BNF) returns true. That would mean that the first input symbol (lookahead) unambiguously determines which branch to follow, and we get back recursive parsing with no backtracking (i.e. predictive parsing) that we used for the BNF definition earlier. Note however, that by the time a procedure returns false, it might have consumed a number of symbols from the input stream. Therefore the input stream pointer must also be stacked along the recursive procedure calls, and each time a rule implemented by its procedure 
is recognised not to be applicable, this pointer has to be restored. This is called backtracking, and there are only a few known parsers using this technique [8], because it's rarely needed for preprocessed context free grammars. But this is the price we pay at the trade off to keep our parser closely follow the untouched standardised set of BNF rules in structure.

\subsection{Synthesis of the SDL parser}

Node types of the abstract syntax tree are generated from the BNF definition of SDL according to decisions explained earlier. Roughly stated, there is a node type for each nonterminal symbol of the BNF definition. For an example, Figure 4 shows the simplified node type "textual system definition" of the abstract syntax tree, that was generated from rule <textual system definition $>$ of the original BNF definiton.

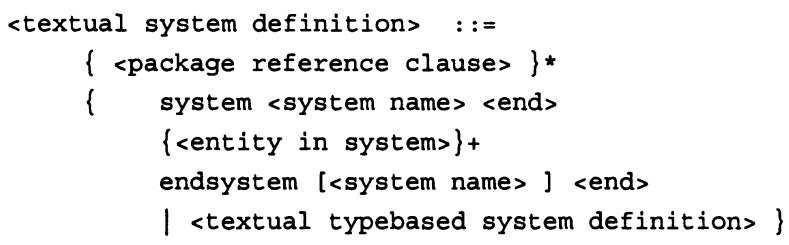

Modelling rules in Z.100 define semantic equivalence of possibly infinite sets of syntax units. Modelling rules and semantic constraints in Z.100 are given with local scope on syntax units defined by individual BNF rules. Therefore it is straightforward to check and enforce them based on an abstract syntax tree of our kind as opposed to one that the "Abstract grammar" definitions in Z.100 would imply. Besides constructing the tree builder functionality from SDL-PR at the synthesis of the SDL parser, these constraints must be implemented manually (remember that they are given informally) as so-called semantic subroutines [2] and put into the synthesis process. Semantic subroutines manipulate on the abstract syntax tree, that will be the basis of Java code generation according to concepts of the Specification domain explained in Section 3. 


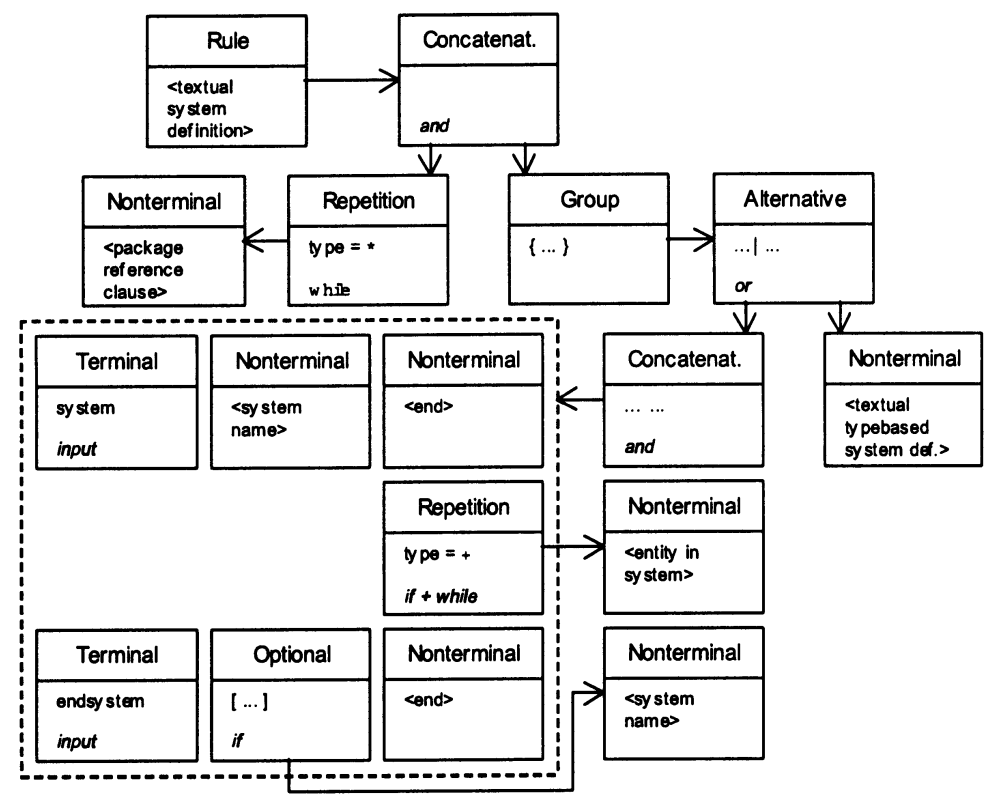

Figure 4. Generated node type for rule "textual system definition"

Whenever a procedure associated with a nonterminal is called, a new instance of the corresponding node type is created, linked to the abstract syntax tree, and all its branches is attempted to parse in recursively. The problem of backtracking of course also applies to these branches of the growing tree. After successful parsing, the derivation tree for the SDL-PR source is given by either the sequence of processes returning true or the abstract syntax tree. The same does not stand for unsuccessful parsing. If the input is not a valid sentence of the SDL language, from one moment in the parsing process all procedures will return false until the last, the one corresponding to the root symbol of the grammar, will also return false. We get the information that the source is syntactically incorrect, but the input stream and the tree were backtracked during the false procedure returns. To get the information how far the parsing process could successfully reach into the input stream, a special algorithm was developed to track the longest possible prefix of the sentence believed to be valid prefix of a valid sentence. Note, that the problem is that by the time the parser gets known about the invalidity of the sentence, the erroneous position, lexical unit (token), and the tree is rewound already by the backtracking. Therefore the algorithm delays the rewinding of the stream with some remembered backtracking parameters. 
Let 'maxpos' be the end marker of the longest prefix believed so far and 'revertpos' be the earliest position in the stream until we had to rewind the input during backtracks. ' $t$ ' will denote time, and pos $(t)$ is our current position of the input stream in the backtracking recursive descent parsing process. In the beginning of parsing Maxpos $=$ revertpos $=0$. Executing the following instructions after each step of the parsing implements the algorithm illustrated in Figure 5.
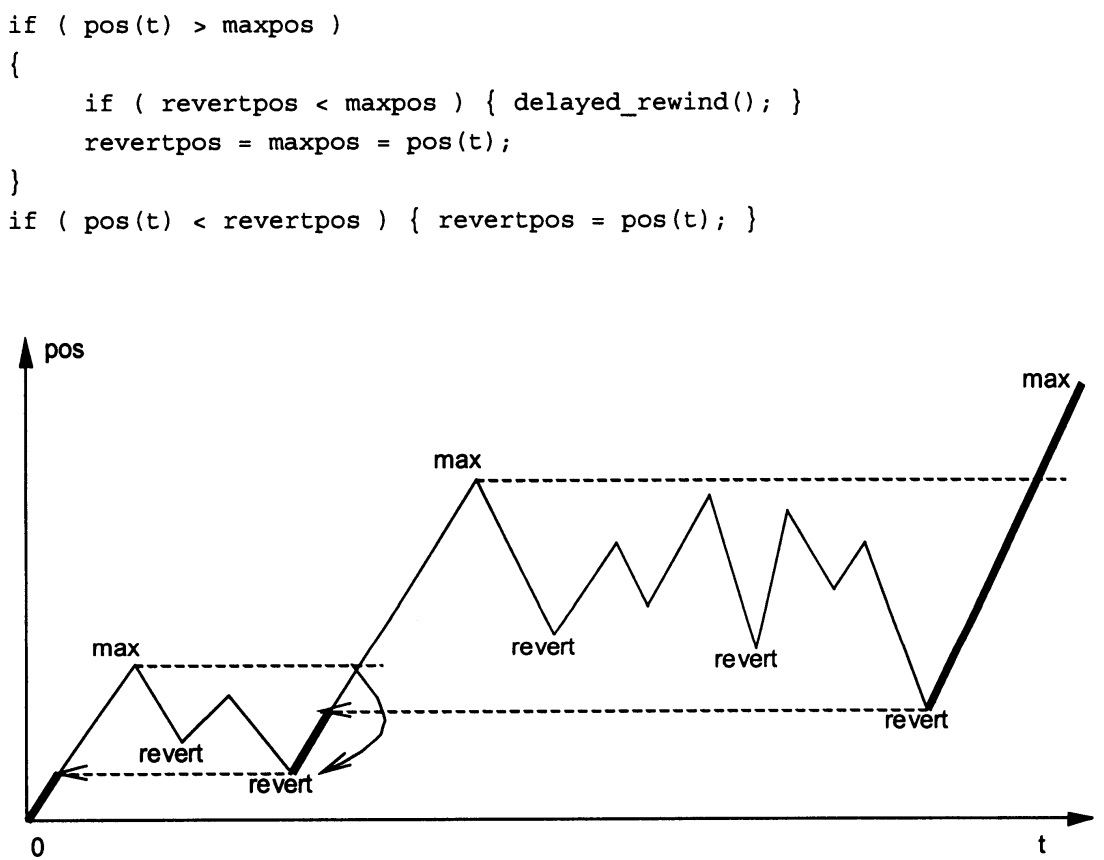

Figure 5. Backtracking parameters for delayed rewinding

\section{SEMANTIC INTERPRETATION MODEL}

In the specification domain Z.100 introduces the concept of named entities. A named entity is either a type or an instance. Processes and signals are examples of types, system, blocks, and variables are examples of instances. The difference is illustrated in [6] as "In general, we can identify instances by the fact that they are bound to a specific place within the system, whereas a type may be used in more than one place". System here 
clearly relates to the specification domain. By the distinction between specification and instance domains, a more precise definition can be formulated. The difference can roughly be defined as the relation between the entity that is given name in the specification domain, and the existence it possibly corresponds to in the instance domain. If this relation is one-tomany, than the named entity is a type, if one-to-one, than it is an instance.

Decisions had to be made concerning the selection of Java constructs for the specification domain (how the original SDL specification phrases are translated), and the instance domain (how the dynamic semantics of SDL comes to life). The decisions for the two domains are of course not independent, and priority was given to the specification domain mapping to achieve an ideal one-to-one readability of the SDL phrases in the generated Java source code. This approach is new and does not follow the transformation rules given by Annex F of Z.100, as will be explained shortly. However, it would enable easy manipulation of the Java code according to SDL terms, therefore this approach fits research oriented, experimenting, prototyping applications most. The accepted trade-off is, that this decision implies hardest requirements on the Java solutions for the instance domain, since all features that could have been handled by manipulation of the abstract syntax tree (or in the specification domain), must be implemented there. The overall process of the prototyping of SDL specifications is illustrated in Figure 1.

\subsection{Formal definition of SDL semantics}

Z.100 gives formal definition of SDL semantics in Annex F. F.1 is introduction to the Meta-IV language that is used in F.2 and F.3 describing the static and dynamic semantics respectively. Their relation is illustrated by the top segment of Figure 6 along with the AS0 and AS1 syntaxes introduced by Z.100. AS0 is not formally defined in the recommendation, but can be derived from the BNF syntax. A constructive example was given to this as a generated abstract syntax tree in Section 2. AS1 is given in Z.100 as the Abstract Grammar. However, with the F.2 transformation from the abstract syntax (representing concrete textual syntax) to AS1 (input to the dynamic behaviour definition of SDL), specification domain information is lost. Such information would regard type inheritance hierarchies or virtuality. Losing this information causes no problem when interpreting SDL, because it is object oriented only in the specification domain, i.e. does not feature polymorphism or means of late binding. 


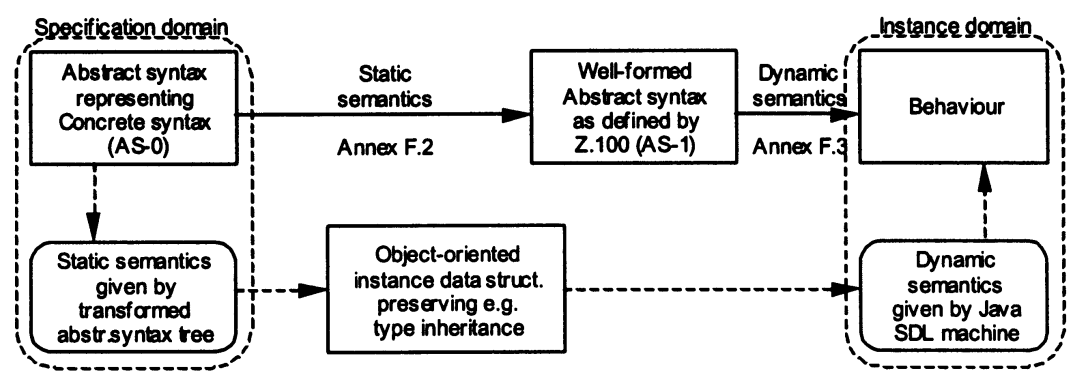

Figure 6. Alternative to Annex F derivation of SDL behaviour

For the same reason one should have wide knowledge in principle about these transformations to produce quality specifications reflecting exactly his/her intentions. For example even though transitions of asterisk states in virtual process types are transformed into transitions for each states of the process type, its asterisk semantics shall also apply to brand new states introduced by redefined process types down the inheritance hierarchy.

In other words, there is a gap between specification domain concepts and instance domain representation, which the designer should be aware of. Our framework attempts to narrow this gap on the price of preserving specification domain concepts for an interpretation with heavier runtime overhead, e.g. using delegation to resolve added and redefined virtual properties down the type inheritance hierarchy. The bottom segment of Figure 6 illustrates this alternative way to derive dynamic behaviour of an SDL specification with the representation forming the basis of behaviour interpretation being closer to the specification domain.

\subsection{Process types and process instances}

Basically each named entity in SDL is mapped to its own Java class. Classes for SDL instances will be inner classes of their corresponding SDL type's Java class. In this manner local management of instance life-cycle, and clear structure of Java specification is achieved.

Taken SDL processes as an example, process Monitor of block Game in Figure 7 will be mapped to the following Java construct:

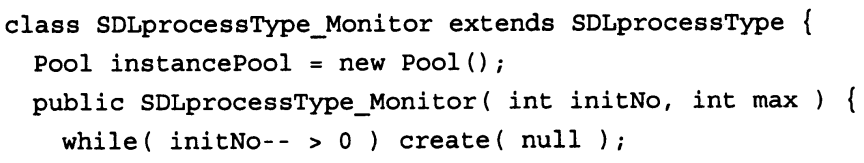



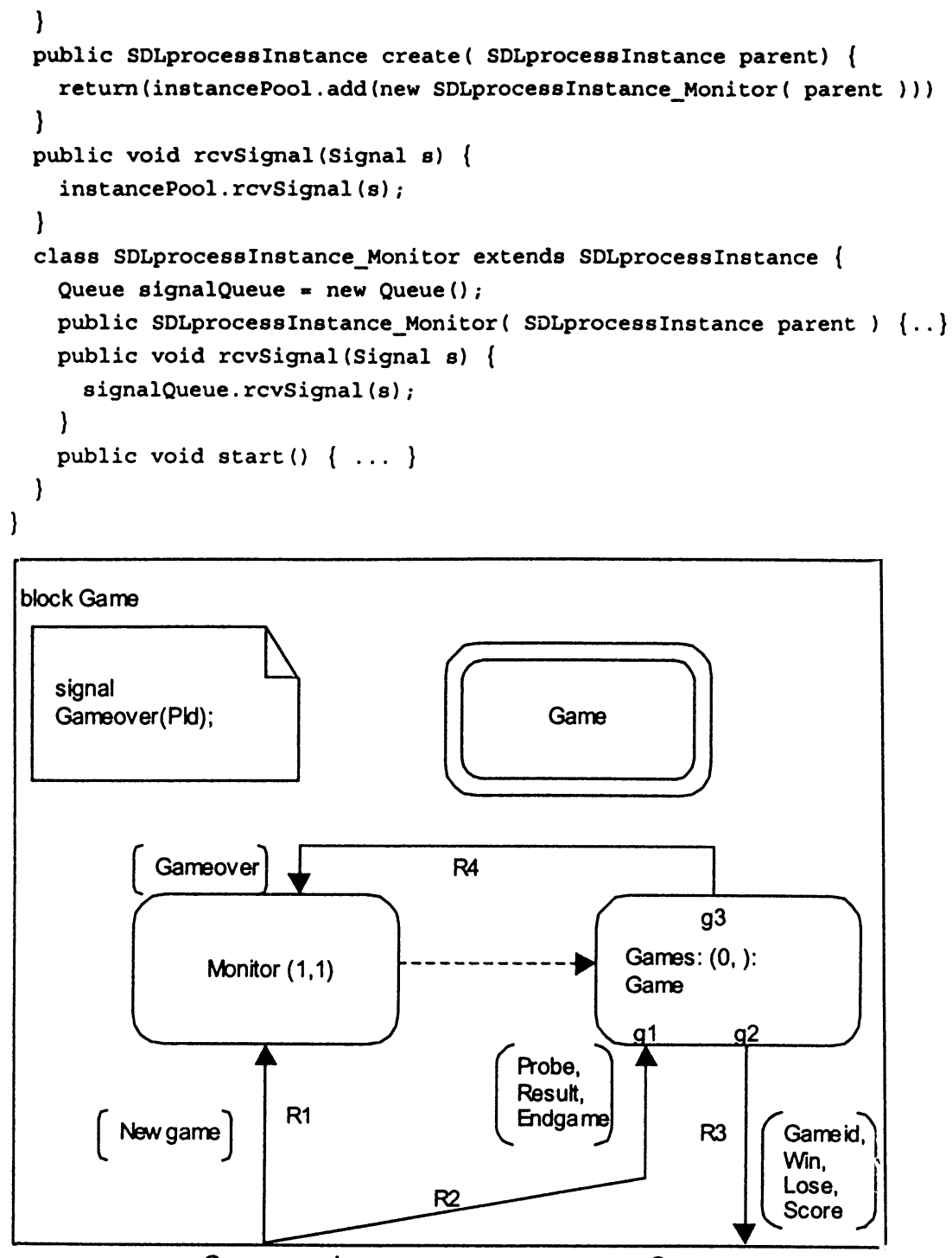

Gameserver.in

Gameserver.out

Figure 7. Example SDL block definition

Process types will create instances of their own type and will deliver incoming signals in case of output-via type signal sending. New to the 
mapping applied by this project that it allows generic context parameterisation and type inheritance to appear in the instance data structure of the instance domain.

\subsection{Signals}

Signals are mapped to an abstract class having a pointer to an array of objects as the list of parameters they can carry. Each signal in the SDL specification will inherit from this abstract class. Methods handling signals identify them by their class type that is given by the Java RTTI (runtime type identification) construct Object.class. Classes responsible for input part of transitions will have a field containing the class type of the input signal they act upon. This mapping enables signal types (i.e. signals having formal context parameters) to be handled the same way signal instances are handled.

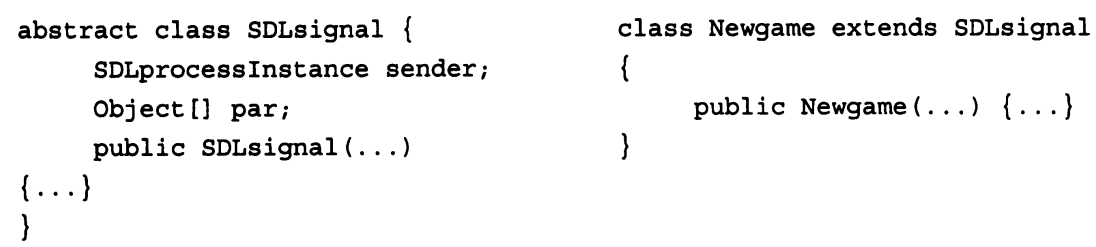

\subsection{Instance domain}

Figure 8 shows the most important classes and their inheritance relations representing SDL named elements for the instance domain. Path is the abstraction of the common properties of named elements that can carry signals in SDL. Plug represents connection points of these paths, typically on the bounderies of blocks, processes, services, and corresponding types. The restriction that connection points on boundaries can only be 1-to-many was removed because with the introduction of types, gates and specialization it was found to be too restrictive. Because the interpretation model applied by the project preserves types for the instance domain, connection points are allowed to be many-to-many. Process types and process instance types reflect the structure explained above.

The following example illustrates the Java skeleton of the runtime system (RTS) with a simple scheduling algorithm. With this algorithm it is possible to fire one armed transition at a time, including spontaneous transitions, continuous and priority signals, and expired timers. Responsibility of checking the armed property of transitions is delegated down the hierarchy of simulation environment, the RTS, processes, their states, and transitions 
of any kind of these states. This mechanism enables parameterised types to be brought from the abstract syntax of the specification domain into the instance data structure of the instance domain. Another advantage is the scheduler's global information about the armed state of the SDL machine, i.e. the disjunction of armed states of all process instances in the system instance. Different interpretation models of e.g. time semantics of SDL can be implemented with specialisation of this RTS class in the object oriented semantic interpretation model.

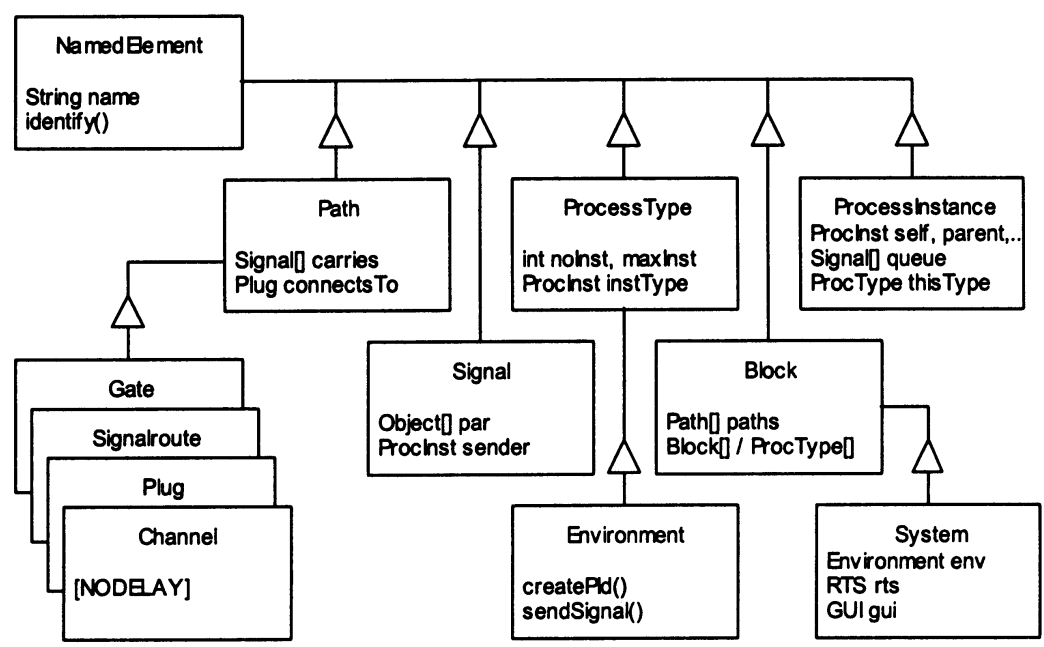

Figure 8. Classes and inheritance relations of the instance domain

The scheduler below fires the first armed transition from a list of active processes, and schedules the fired process into the end of the list. An interpretation generating a larger set of possible traces can be implemented by using 'any' instead of 'first' in the algorithm below.

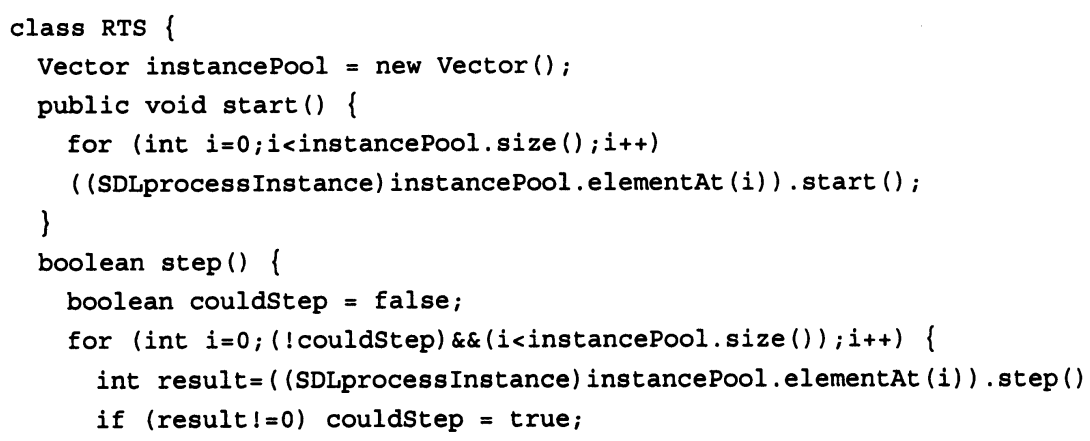




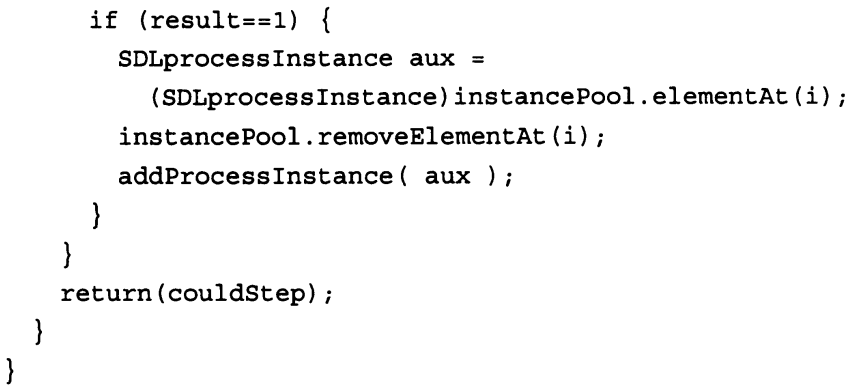

\subsection{A new approach to time semantics in SDL}

Due to the current time semantics of SDL, time may or may not be advanced by transitions and transactions during system interpretation. Figure 9 illustrates an approach where the chosen interpretation of time semantics is orthogonal to the scheduler algorithm above. This solution is compatible to different interpretations of time semantics with respect to the generated set of possible traces. Whenever a process instance encounters setting of a timer, it is interpreted as sending a signal to a global timer process with parameters of signal name, sender id, and expiration time. The timer process's queue is sorted by these expiration time values, and handled by the scheduler the same way other processes are handled. Upon interpreting the reception of a signal by the timer process, it sets the global simulation time to the specified expiration time parameter, and sends a signal with the specified signal name parameter to the specified sender parameter. If the timer process is always scheduled last among all processes, the interpretation will generate a trace set equivalent to the "time may not be advanced..." interpretation. If the scheduler randomly selects armed processes including this timer process, the resulting set of possible traces will be equivalent to the "may not be advanced..." interpretation. Note, that with this separation and orthogonal modelling of semantic interpretation functions, different concepts can be experimented with by prototyping in the modular object-oriented framework of semantic interpretation models. 


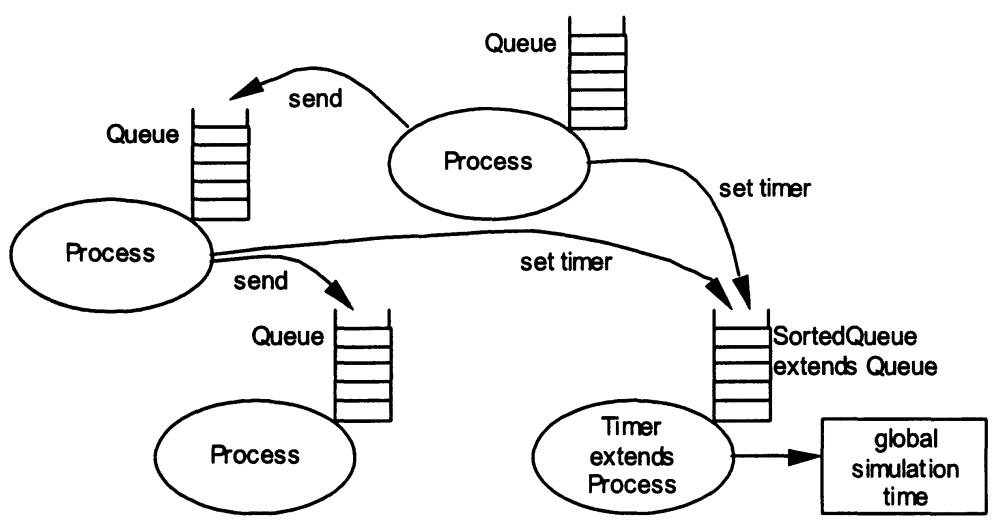

Figure 9. Time semantics interpreted with global timer process approach

\section{APPLICATION TO PERFORMANCE EVALUATION}

\subsection{PerfSDL, protocol performance modelling in SDL}

Covering timing properties and identifying performance bottlenecks in testing of proprietary protocols is one method to relate protocol performance to system performance. Because an SDL system is not limited by processing resources (i.e. assumed to be fast enough to process any load it is offered), it would be cumbersome to assess performance measures by means of modifying protocol specifications with available SDL constructs. Instead, PerfSDL [10] describes protocol performance integrated into system performance, where new SDL constructs provide appropriate correlation and feedback. These new constructs, illustrated in Figure 10, imply modifications to the BNF definition of the concrete grammar, to the semantic actions on the abstract syntax tree, and to the Java implementation of the meta-process "SDL-machine". In the concrete grammar, the production rule for <action $>$ has been extended with <timed transition $>$ and $<$ probe>, and production rules for these have been defined. After automatic generation of the parser and AS0 abstract syntax tree, the semantic actions are systematically included. Implementation of the dynamic behaviour of the constructs involves a new layer to the SDL-machine that manages system time and collects information during simulation. This information can be 
analysed off-line, and results can be fed back to subsequent iterations of the protocol development process.

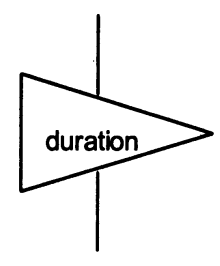

timed-transition

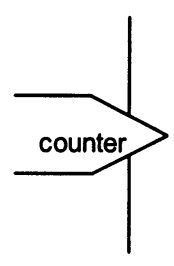

probe

Figure 10. Introduction of new constructs for performance testing in [10]

\subsection{First experiences applying the framework to a small example}

Two simple packet transfer protocol alternatives were specified in PerfSDL using the new constructs, and two special SDL processes. One generated system stimuli as packets to be sent according to different stochastic arrival processes. Information relevant to protocol performance was sent to the other, an observer process, as signal parameters. These signals were monitored and logged, and later evaluated. Based on the evaluation, performance of the protocol alternatives was compared under different workload conditions.

\section{CONCLUSIONS}

A prototyping framework for SDL protocol specifications with extended and modified SDL semantics was introduced based on automated parser and abstract syntax tree generation and an open object-oriented semantic interpretation model using Java technology. The framework supports experimenting with SDL specifications of extended semantic foundations. A systematic approach was applied for treating the effects of semantic extensions on different phases of the prototyping process. A new general interpretation model of time semantics was described that is orthogonal to other semantic interpretation functions and can be specialised for modelling different actual interpretations of SDL's time semantics. Application of early 
implementation of the prototyping framework was shown in the field of SDL-based protocol performance engineering.

\section{ACKNOWLEDGEMENTS}

The author is thankful to Miklós Boda for the support of Traffic Lab at Ericsson Telecommunications, where the project has been carried out. At the Technical University of Budapest Professor Gyula Csopaki and Professor Iván Bach gave support and helpful instructions. Thanks to Professor Finn Arve Aagesen for commenting the paper and for the supervision throughout the years at the Department of Telematics, Norwegian University of Science and Technology.

\section{REFERENCES}

1. Ferenc Belina, Dieter Hogrefe, Amardeo Sarma, SDL with Applications from Protocol Specification, Prentice Hall International, 1991

2. Ivan Bach, Computer Linguistics, lecture notes, 1991

3. Rolv Bræk, Øystein Haugen, Engineering Real Time Systems, Prentice Hall International, 1993

4. Anders Olsen, Ove Færgemand, Birger Møller-Pedersen, Rick Reed, J.R.W. Smith, Systems Engineering Using SDL-92, Elsevier, 1994

5. ITU-T Recommendation Z.100, CCITT Specification and Description Language (SDL) and Annex F. Static and Dynamic Semantics of SDL, 03/93, International Telecommunication Union, 1995

6. Jan Ellsberger, Dieter Hogrefe, Amardeo Sarma, SDL Formal Object-oriented Language for Communicating Systems, Prentice Hall Europe, 1997

7. Øystein Haugen, Birger Møller-Pedersen, SDL - a platform for telecom system development also in the future?, Ericsson and Telelogic Joint User Group Conference 1998

8. Alfred V. Aho, Ravi Sethi, Jeffrey D.Ullman, Compilers Principles, Techniques, and Tools, Addison Wesley, March 1998

9. Olivier Dedieu, A Dynamically Extensible Web Proxy, NoTeRe colloquium, 20-23 October 1998. 
10. Mazen Malek, PerfSDL: an Interface to Protocol Performance Analysis by means of Simulation, Accepted paper, $9^{\text {th }}$ SDL Forum, Montreal, June 1999

11. R.Gotzhein, B.Geppert, F.Rossler, P.Schaible, Towards a New Formal SDL Semantics, SAM98, $1^{\text {st }}$ SDL and MSC workshop of the SDL Forum Society, Berlin, July 1998

12. Andreas Mitschele-Thiel, Performance Evaluation of SDL Systems, SAM98, $1^{\text {st }}$ SDL and MSC workshop of the SDL Forum Society, Berlin, July 1998

13. E.Holz, J.Fischer, Introduction of Gate Types into SDL, SAM98, $1^{\text {st }}$ SDL and MSC workshop of the SDL Forum Society, Berlin, July 1998

14. Andreas Mitschele-Thiel, Results of the discussion, Workshop on Performance and Time in SDL and MSC, Erlangen, February 1998 